\title{
SVD-EBP Algorithm for Iris Pattern Recognition
}

\author{
Mr. Babasaheb G. Patil \\ Department of Electronics Engineering \\ Walchand College of Engineering, \\ Sangli, (Maharashtra) India
}

\author{
Dr. Mrs. Shaila Subbaraman \\ Department of Electronics Engineering \\ Walchand College of Engineering, \\ Sangli, (Maharashtra) India
}

\begin{abstract}
This paper proposes a neural network approach based on Error Back Propagation (EBP) for classification of different eye images. To reduce the complexity of layered neural network the dimensions of input vectors are optimized using Singular Value Decomposition (SVD). The main objective of this work is to prove usefulness of SVD to form a compact set of features for classification by EBP algorithm. The results of our work indicate that optimum classification values are obtained with SVD dimensions of 20 and maximum number of classes as 9 with the state-of-the art computational resources The details of this combined system named as SVD-EBP system for Iris pattern recognition and the results thereof are presented in this paper.
\end{abstract}

Keywords- Singular value decomposition (SVD); Error back Propagation (EBP).

\section{INTRODUCTION}

Biometrics is one of the areas of research that has gained widespread acceptance in the field of human identification and fraud prevention. Although the current state-of-the-art provides reliable automatic recognition of biometric features, the field is not completely researched. Different biometric features offer different degrees of reliability and performance. The Human iris is one of the biometric parameters of the human body efficiently used for person recognition. Number of researchers has worked on person identification using iris as biometric [1, 2 , and 3]. However the results obtained by them indicate strong dependence of recognition/classification accuracy on the orientation of the iris image as well as on the light intensity levels while capturing images.

This paper confirms the usefulness of Singular Value Decomposition method- SVD (as was recommended by [4]) to extract a compact set of features from the preprocessed iris image to overcome the earlier drawback of orientation and intensity dependent classification accuracy for iris recognition.

\section{A. Characteristics of Human Iris [4]}

The use of the human iris as a biometric feature offers many advantages over other human biometric features. The Iris is the only internal human body organ that is visible from the outside and is well protected from external modifiers. A fingerprint, for example, may suffer transformations due to harm or aging, voice patterns may be altered due to vocal diseases. However, the human iris image is relatively simple to acquire and may be done so in a non-intrusive way. The Human iris starts forming right from the third month of gestation in the mother's uterus. A small part of final iris pattern is developed from the individual DNA while most of the part is developed randomly by the growth of epithelial tissues present there. It means that two eyes from the same individual, although they look very similar, have two different patterns of two Irises, however with unique DNA related internal pattern. Identical twins would then exhibit four different iris patterns and can be uniquely recognized using these patterns. Hence human identification using iris images has become a favorite choice among researchers in recent years.

\section{GENERIC PROCESS FLOW}

A generic process flow for iris pattern recognition and classification is as shown in Figure 1. As it is clear from the figure, the entire process flow broadly consists of two parts. The first part corresponds to image pre-processing in order to extract an optimal and compact set of features while the second part deals with pattern recognition and classification. In our research work a Singular Value Decomposition (SVD) method was used to extract a compact set of features on the preprocessed iris images where the pre-processing was carried out using standard steps viz. image acquisition, image segmentation, edge detection etc.

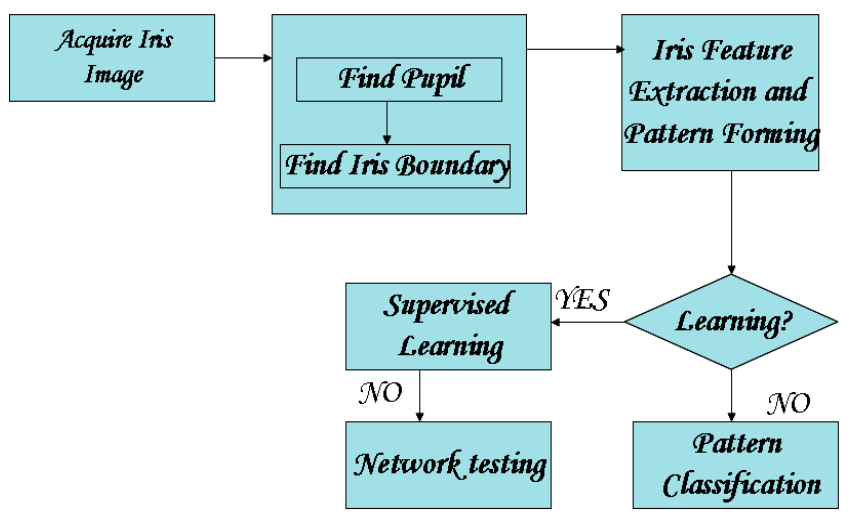

Figure.1: Processing Steps

In the second part, these features were inputted to multilayered neural network classifier implementing Error Back Propagation (EBP) algorithm. EBP is a supervisory algorithm generally used in two phases' viz. training phase and testing phase. MATLAB was extensively used in the entire research work. The details of preprocessing are given in the next section while the details of recognition and classification are presented in the Section IV of the paper. The conclusions derived from this work are presented in the Section V highlighting the future scope in this area. 


\section{PREPROCESSING}

As stated above, pre-processing contains following three steps $[6,7]$

- Image Acquisition

- Image Segmentation and Edge Detection

- Feature Extraction

The details of these steps are given below.

\section{A. Image Acquisition}

The major problem of iris Recognition using already researched methods is image acquisition because of the susceptibility of eyes to degree of illumination. The importance of the image grabbing system implementing consistent illumination has been spelled out in literature. But the approaches of SVD-EBP used by the authors of this paper do not pose any such restriction on the intensity level of light illumination. However, the pupil is an open door to the retina, one of the most sensitive organs of our body, and extra care must be taken when shedding direct light on it.

The work presented in this paper uses the CASIA iris database as input.[8] This database uses a special camera that operates in the infrared spectrum of light, not visible by the human eye. Here, each iris class is composed of 7 samples taken in two sessions, three in the first session and four in the second session. The two sessions were taken with an interval of one month. Images are $320 \times 280$ pixels gray scale taken by a digital optical sensor designed by NLPR (National Laboratory of Pattern Recognition Chinese Academy of Sciences). There are 108 classes or total number of iris images is 756 . Figure 2 shows a sample of the image of an eye from this database.

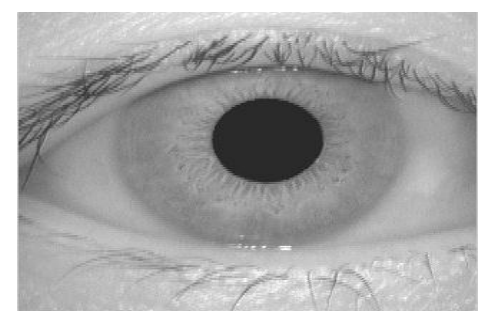

Figure.2. Image of an Eye

\section{B. Iris Segmentation}

The main motive behind iris Segmentation is to remove the non-useful information like the sclera and the pupil information and extract the region of interest. First, the pupil is detected and then the iris-sclera boundary is detected which is usually done in two steps B1 and B2 as given below [4].

\section{1) Detection of Pupillary Boundary}

As we all know that pupil is a very dark blob of a certain minimum size in the picture and no other segment of continuous dark pixels is of the same size. This makes an easy task to detect the pupillary boundary in the iris image. This algorithm finds the center of the pupil and two radial coefficients as the pupil is not always a perfect circle.

To find the pupil, we first need to apply a step threshold to the image given by,

$$
\begin{array}{r}
g(x)=f(x)>70: 1 \\
f(x) \leq 70: 0
\end{array}
$$

where $f(x)$ is the original image and $g(x)$ is the threshold image. Pixels with intensity greater than the empirical value of 70 (in a 0 to 255 scale) are dark pixels, therefore converted to 1 (white). Pixels with intensity smaller than or equal to 70 are assigned to 0 (black). Figure 3 shows the threshold image of the pupil. Since, the eyelashes also satisfy the threshold condition, they are visible in the figure.

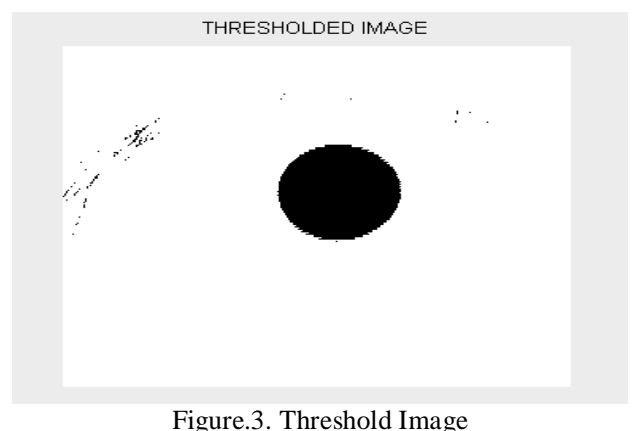

To eliminate the area of eyelashes, search of a region of 8 connected pixels with value 1 is carried out. The report on CASIA database indicates that an area value of 2500 is sufficient for pupil region. The eyelashes definitely have much smaller region than the pupil region, hence the area associated with eyelashes would be much smaller than 2500 . Using this knowledge, one can cycle through all regions and apply the following condition:

$$
\begin{gathered}
\text { for each Region } R \\
\text { if } A R E A(R)<2500 \\
\text { set all pixels of } R \text { to } 0
\end{gathered}
$$

Thus the pupil is separated and the centroid $\left(\mathrm{x}_{\mathrm{cp}}, \mathrm{y}_{\mathrm{cp}}\right)$ of the pupil is extracted. Also horizontal and vertical radii are calculated. Figure 4 shows the threshold image in which the eyelashes have been cropped out by Freeman's Chain Coding method [4].

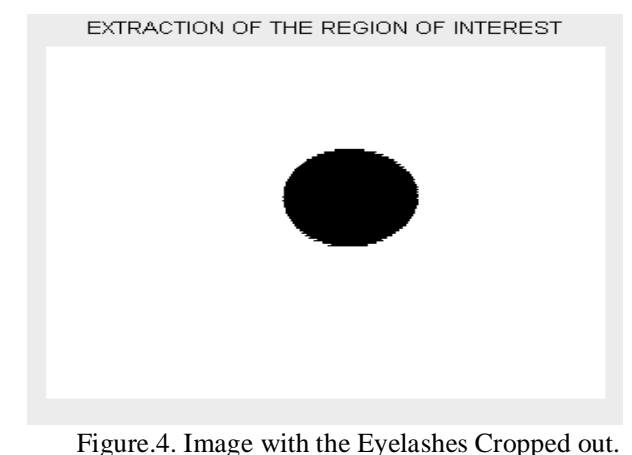

Figure.4. Image with the Eyelashes Cropped out.

\section{2) Iris Edge Detection}

Figure.5 shows the pupil in the original eye image with centroid $\left(\mathrm{x}_{\mathrm{cp}} \mathrm{y}_{\mathrm{cp}}\right)$, horizontal radius $\mathrm{r}_{\mathrm{x}}$ and vertical radius $\mathrm{r}_{\mathrm{y}}$.

After detecting the pupil the next step is to find the contour of the iris. Already, we have detected the pupil location and we have the knowledge that it is concentric to the outer perimeter of the iris. 


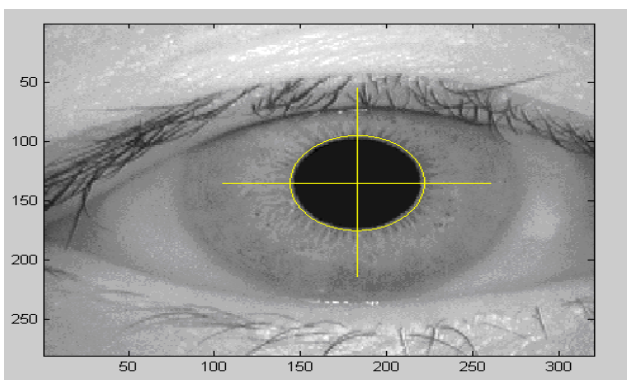

Figure.5 . Detection of the centroid $\left(\mathrm{x}_{\mathrm{cp},} \mathrm{y}_{\mathrm{cp}}\right)$ and horizontal radius $\mathrm{r}_{\mathrm{x}}$ and vertical radius $r_{\mathrm{y}}$

But, the problem is that sometimes the eyelid may occlude part of the iris. Also, the iris center may not match with the pupil center, and we will have to deal with strips of iris of different width around the pupil. This method takes into consideration the fact that areas of the iris at the right and left side of the pupil contain the most significant information that is useful for data extraction. The areas above and below the pupil carry unique information, but it is very common that they are totally or partially occluded by eyelashes or eyelids.

Figure 6 shows the steps how to find the right edge of the iris. The strategy adopted for iris detection is to trace a horizontal imaginary line that crosses the whole image passing through the center of the pupil [4]. This figure shows the horizontal line passing through $\mathrm{y}_{\mathrm{cp}}$ (center of pupil) of original image. Corresponding to pixels on this line, the pixel intensities are also shown in the figure graphically.

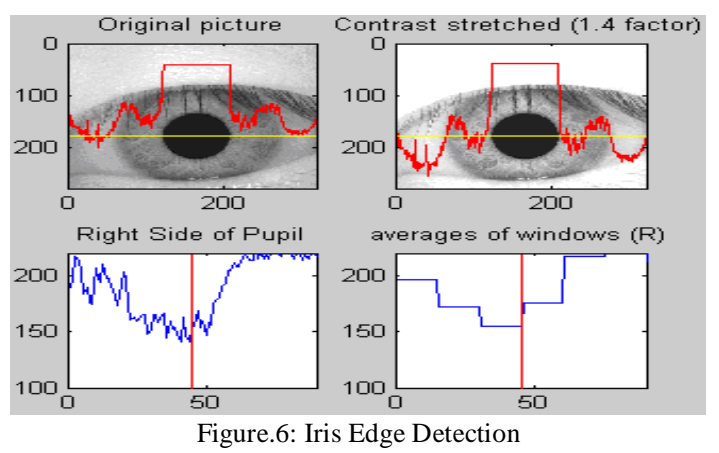

Starting from the edges of the pupil, we analyze the signal composed by pixel intensity from the center of the image towards the border and try to detect abrupt increases of intensity level. Although the edge between the iris disk and the sclera is most of the times smooth, it is known that it always has greater intensity than iris pixels. We intensify this difference applying a linear contrast filter. It is possible that some pixels inside the iris disk are very bright, causing a sudden rise in intensity. That could mislead the algorithm to detect it as an iris edge at that point. To avoid this, we take the average intensity of small windows when the sudden rises occur from these intervals as shown in Figure 6.

\section{Feature Extraction}

Once the segmentation has been performed and the region of interest (ROI) has been extracted, the next step is to extract the features so as to reduce the problem of dimensionality. The iris Basis Images are extracted by converting polar information of the iris into Cartesian information with radial resolution of 10 pixels and specific angular resolution. in terms of pixel count (Y-axis) for angles varying from $0^{0}$ to $360^{\circ}$ (X-axis) in specific steps. Pixels on either side of the pupil are collected and one reduced image of the iris is formed. Thus, the extraction of iris basis reduces the dimension as the non-useful information is cropped out. We can better visualize this strategy by looking at Figure 7 as shown below.

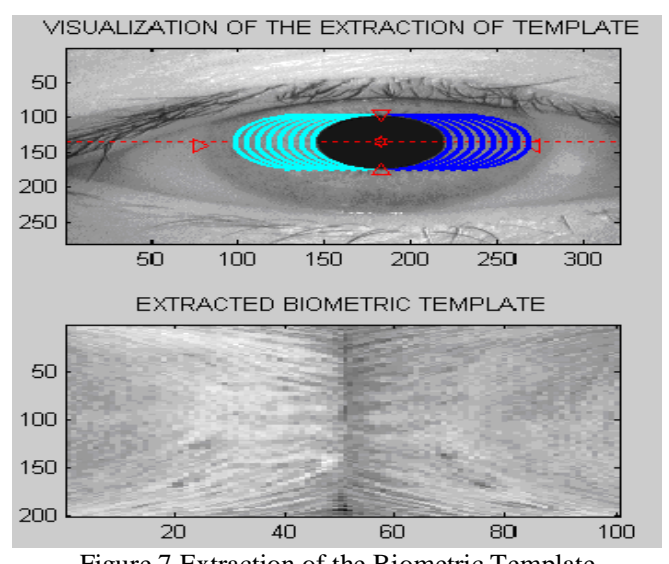

Figure.7 Extraction of the Biometric Template

Up-till now though the dimension has been reduced significantly, it is still too high for classification. So, the singular value decomposition, as explained below, is employed for further reduction in dimension.

\section{1) Singular Value Decomposition (SVD)}

Singular Value Decomposition is a powerful tool in matrix computations and analyses which has the advantage of being quite robust to numerical errors $[9,10]$. Additionally SVD exposes the geometric structure of full-rank matrix. The presence of noise in image, either from image capturing systems or from round-off numerical errors, results into an image matrix that is generally of full rank. Hence SVD is a recommended technique to decompose the data into an optimal estimate of signal and the noise components. Further SVD aids in image compression by storing the image information in only $M$ elements of a MXN image matrix $(M \geq N)$. For all these reasons, we have used SVD in deriving a compact and representative set of features of the iris images grabbed even with light intensity variation and in presence of noise.

The basic operation of SVD relies on the factorization of an MxN matrix $(M \geq N)$ into three other matrices on the following form:

$$
A=U^{T} \delta V
$$

where the superscript " $T$ " denotes transpose. $U$ is an MxM orthogonal matrix, $V$ is an NxN orthogonal matrix and $\delta$ is an MxN diagonal matrix with $s_{i j}=0$ if $i \neq j$ and $s_{i i} \geq s_{i+1, i+1}$. The two important aspects to be noted here are:

1. $\delta$ is zero everywhere except in the main diagonal. This leads to reduction in the dimension of the input pattern from a matrix $\mathrm{MxN}$ to only a vector of $\mathrm{N}$ elements.

2. Only the first few elements contain substantial information, and the vector tail without significant loss of information can be cropped out. 
Figure 8 shows a plot of SVD pattern vectors which very well depicts the second property mentioned above.

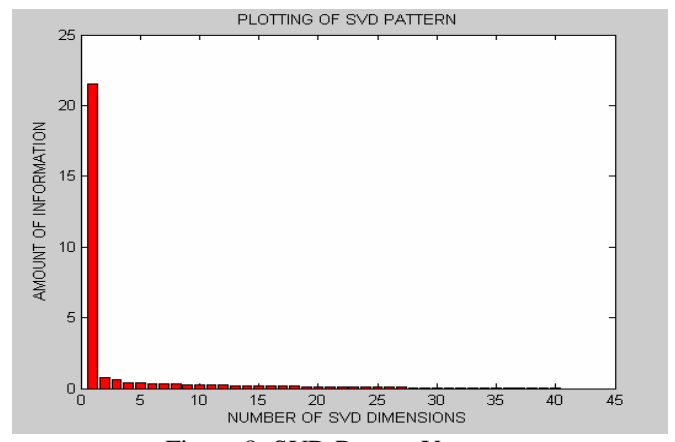

Figure.8: SVD Pattern Vectors.

\section{ClassificATION}

As mentioned in the Generic Process Flow, second part of this paper deals with iris classification. Here, the SVD features extracted as explained above were inputted to multilayered neural network classifier implementing Error Back Propagation (EBP) algorithm. Here, any five patterns of total 7 patterns of each of the 108 classes of CASIA database were used for network training and the remaining two patterns of each of 108 classes were used for network testing. Our network implements the classical 3-layer architecture: Input layer, Hidden layer and Output layer. The input layer contains as much neurons as the dimensionality of the pattern vector, which we have limited to 10 (Refer Figure 8) in the present case. The number of neurons in the hidden layer is approximately double as that of input layer (i.e. 20 ) for good classification results. The number of neurons in the output layer corresponds to the number of classes to be recognized.

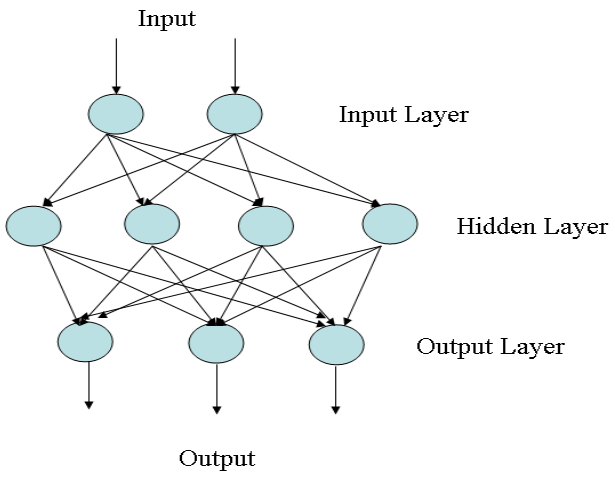

Figure.9. Architecture of Feed Forward Back propagation Neural Network

\section{A. Network Design}

Following are some parameters set for network training in MATLAB

- Training function: traingda (Adaptive learning rate)

- Initial learning rate: 0.2

- Learning rate increment: 1.05

- Maximum Epochs: 50,000

- Error goal: $5 \times 10^{-7}$

- Minimum gradient: $1 \times 10^{-9}$

The various experiments performed to train the neural network and test the iris pattern include iris-basis images of
$40 * 40$ pixels quantized from the original iris image with a mask of $3 * 3$ pixels. The SVD algorithm as discussed in the section III of this paper was developed to output the vectors with $3,10,20$ and 40 dimensions. The target classes for classification were varied from 3 to 20 .

When the network was trained in the supervised mode, a target vector was also presented to the network. This target vector has every element set to zero, except on the position of the target class that will be set to 1 . The idea behind this design decision is that for each input pattern $\mathrm{X}$ presented to the network, an output vector $\mathrm{Y}$ is produced. This vector has the number of elements equal to numbers of output neurons. Each output neuron implements a squashing function that produces a real number in the range $(0,1)$. To determine which class is being indicated by the network, we select the maximum number in $\mathrm{Y}$ and set it to 1, while setting all other elements to zero. The element set to one indicates the classification of that input pattern. Figure 10 shows the convergence behavior of neural network using Gradient Descent algorithm for a typical case as obtained from MATLAB. It is seen that the MSE was reached within 2150 epochs.

While simulating using MATLAB, it was found that as the number of classes increased, the network had more difficulty in learning the proper discriminatory weights. The network was able to reach the MSE goal within the specified number of epochs. For number of classes > 6, the MSE goal was not attained anymore, but the MSE kept decreasing until the maximum number of epochs was reached. We feel that increasing the number of epochs may allow the network to eventually converge.

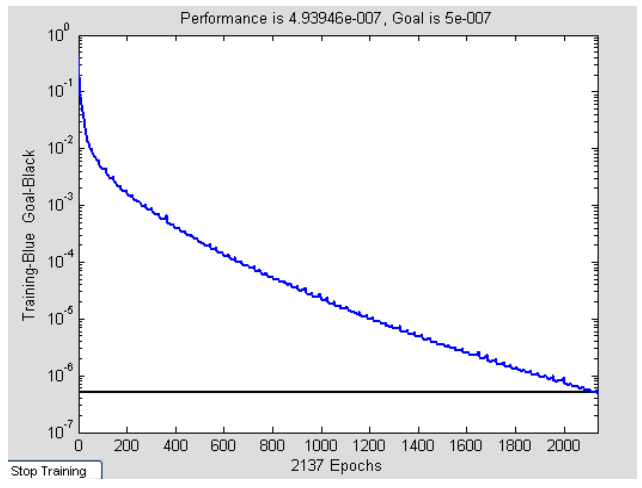

Figure10. Training of the Feed Forward Neural Network

\section{RESUlTS}

Table I shows the classification accuracy with variation in the number of SVD dimensions and number of classes. It is seen from the table that the classification accuracy is $100 \%$ upto five number of classes with requirement of at least 20 SVD dimensions. Increasing these dimensions to 40 does not make significant difference in the classification accuracy. But, it can be seen that as the number of classes are increased beyond 9 the system tends to become over-biased towards one class and the classification accuracy becomes poor even with forty SVD dimensions.

This behavior is also shown in Figure 11. Though the trend of our results using SVD-EBP system is consistent with that of 
[4], the classification accuracies obtained by us for a specific class and specific dimension of SVD vector are better than those reported in this reference. From the results it is seen that this method of classification perhaps cannot be applied for classes above 20. This is due to the fact that with increase in number of classes, a huge computational burden is being exerted on neural network making it incapable to handle voluminous data in-spite of the reduction in the dimensionality of the input vector (representative of 2-D iris image) obtained by SVD approach.

TABLE I. CLASSIFICATION OF DIFFERENT CLASSES USING DIFFERENT DIMENSION

\begin{tabular}{|c|c|c|c|c|}
\hline \multirow{5}{*}{$\begin{array}{c}\text { Number of } \\
\text { Classes }\end{array}$} & \multicolumn{4}{|c|}{ Number of SVD Dimensions } \\
\cline { 2 - 5 } & $3 \mathrm{D}$ & $10 \mathrm{D}$ & $20 \mathrm{D}$ & $40 \mathrm{D}$ \\
\hline 3 & $50 \%$ & $100 \%$ & $100 \%$ & $100 \%$ \\
\hline 4 & $50 \%$ & $87.5 \%$ & $100 \%$ & $100 \%$ \\
\hline 5 & $50 \%$ & $80 \%$ & $100 \%$ & $100 \%$ \\
\hline 6 & $41.66 \%$ & $58.33 \%$ & $91.67 \%$ & $91.67 \%$ \\
\hline 7 & $57.14 \%$ & $64.29 \%$ & $92.86 \%$ & $78.57 \%$ \\
\hline 9 & $61.11 \%$ & $55.55 \%$ & $94.44 \%$ & $83.33 \%$ \\
\hline 10 & $35 \%$ & $55 \%$ & $70 \%$ & $65 \%$ \\
\hline 20 & $27.5 \%$ & $50 \%$ & $55 \%$ & $52.5 \%$ \\
\hline
\end{tabular}

Classification Rate Chart for Patterns Pre-Processed with SVD

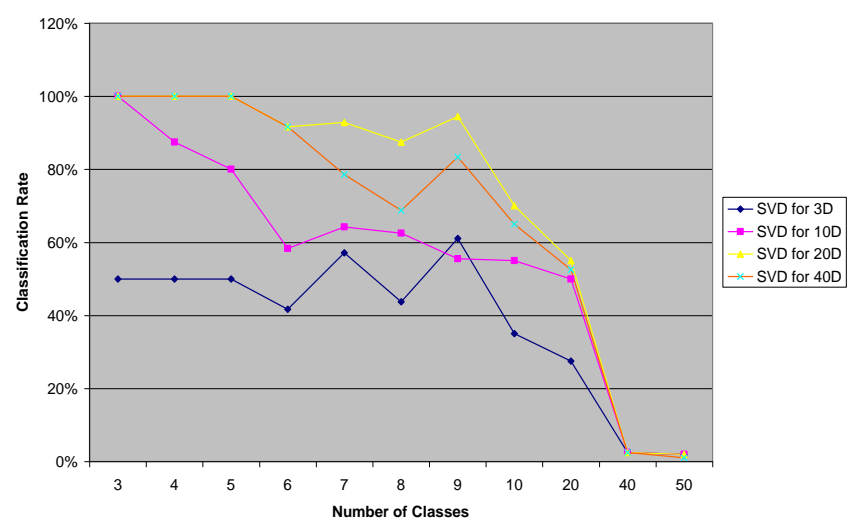

Figure.11 Classification Rate Chart for patterns Pre-Processed with SVD

\section{CONClusion AND Future SCOPE}

A method for pattern recognition based on Singular Value Decomposition (Feature Extraction) with Error Back Propagation of Neural Network (Recognition) was successfully implemented for iris recognition. Number of experiments was carried out with varying SVD dimensions and number of classes. The results of our work indicate that optimum classification values are obtained with SVD dimensions of 20 and maximum number of classes as 9 with the state-of-the art computational resources.

For SVD classes around 20 the performance of the network drops abruptly and becomes independent of SVD vector dimension. This suggests a future scope on researching a better computationally efficient and robust classifier which can handle more number of classes for pattern recognition.

\section{ACKNOWLEDGMENT}

The authors wish to acknowledge Institute of Automation, Chinese Academy of Sciences for making CASIA iris image database freely available on web for carrying out research in this field.

\section{REFERENCES}

[1] Li Ma, Tieniu, Tan, "Personal Identification Based on Iris Texture Analysis", IEEE Trans. On Pattern Analysis and Machine Intelligence, Vol 25, No. 10, Dec. 2003, pg 1519-1533

[2] Daniel Schonberg, darko Kirovski, " Eyeerts", IEEE Trans. On Information Forsensics and Security, Vol 1, No.2, June 2006, Pg 114153]

[3] S.Lim, KLee , .O. Byeon,and T.Kim, "Efficient Iris Recognition Through Improvement of Feature vector and Classifier," Journal of ETRI,Vol 23, No.2 pp. 61-70,2001

[4] Paulo Eduardo Merlotti, "Experiments on Human Iris Recognition using Error Back-Propagation Artificial Neural Network", Project Report, san Diego State University, April 2004

[5] Daugman, J., "How Iris Recognition Works", IEEE Transactions on Circuits and Systems for Video Technology, Vol. 14, Number 1, January 2004.

[6] Kefeng Fan, Qingqi Pei, Wei Mo, Xinhua Zhao, Qifeng Sun, “An efficient Automatic Iris Image Acquision and Pre-Processing System", Proc. 2006 IEEE Conf. on Mechatronics and Automation, June 2006, China, pg 1779-1784

[7] Kittipol Horapong, Jirayut Sreecholpech, Somying Thainimit, Vutipong Areekul, "An Iris Verification using Edge Detection", ID- 07803-92825/05@2005 IEEE, pg 1434-1438

[8] CASIA iris image database, Institute of Automation, Chinese Academy of Sciences, [http://www.sinobiometrics.com]

[9] Louis L. Schart, "The SVD and Reduced-Rank Signal Processing in SVD and Signal Processing II: Algorithms, Applications, and Architectures", Pg 3-31, Elsevier Science Publishers, North Holland, 1991.

[10] Dr. Garcia. E, "Singular Value Decomposition (SVD)- A Fast Track Tutorial", 2006, http:www.misslits.com

[11] Howard. D, Beale. M, Hagon. M, Neural Network Toolbox for use with MATLAB.

\section{Authors Profile}

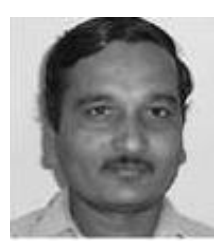

Mr. Babasaheb G. Patil : He received his M.E. Electronics degree in 1990 and B.E. Electronics in 1988. $\mathrm{He}$ is currently working as a associate professor in department of Electronics in Walchand College of Engineering, Sangli, Maharashtra, India. He is having keen interest in image processing and communication. He is carrying out research work in the field of Image Processing.

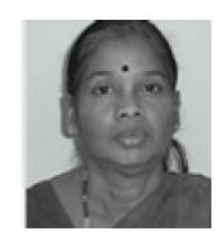

Dr. (Mrs) Shaila Subbaraman : She received M-Tech degree from IISc. Bangalore in 1975 and Ph.D. from IIT Bombay in 1999. She worked in Semiconductor Device Manufacturing company from 1975 to 1989. Currently she is Professor in Department of Electronics in Walchand College of Engineering, Sangli, Maharashtra, India. She has keen interest in the field of Microelectronics and VLSI Design. 